10,04

\title{
Влияние наночастиц на макроскопическую жесткость аморфных тел
}

\author{
() Д.А. Конюх ${ }^{1,2}$, Я.М. Бельтюков ${ }^{2}$, Д.А. Паршин ${ }^{3}$ \\ ${ }^{1}$ Санкт-Петербургский Политехнический университет, \\ Санкт-Петербург, Россия \\ ${ }^{2}$ Физико-технический институт им. А.Ф. Иофффе РАН, \\ Санкт-Петербург, Россия \\ ${ }^{3}$ Санкт-Петербургский академический университет РАН, \\ Санкт-Петербург, Россия \\ E-mail: conyuh.dmitrij@yandex.ru
}

Поступила в Редакцию 27 фревраля 2019 г.

В окончательной редакции 27 фревраля 2019 г.

Принята к публикации 5 марта 2019 г.

\begin{abstract}
В рамках модели случайных матриц, обладающих трансляционной симметрией, изучено влияние нановключений на макроскопическую жесткость аморфных систем. Путем численного анализа получено, что если радиус нановключений $R$ достаточно большой, то применима макроскопическая теория упругости, которая определяет добавку к модулю Юнга $\Delta E \sim R^{3}$. Однако при уменьшении радиуса нановключений эта зависимость становится квадратичной, $\Delta E \sim R^{2}$. Сведением энергии всей системы к сумме квадратичных форм была получена теоретическая оценка модуля Юнга с помощью теоремы Гаусса-Маркова. Из нее следует, что жесткость среды зависит от разности числа связей и числа степеней свободы системы, которая пропорциональна площади поверхности нановключений. Показано, что существует масштаб радиуса нановключений, который характеризует масштаб неоднородности аморфного тела и определяет наименьший характерный размер нановключений, при котором применима макроскопическая теория упругости.
\end{abstract}

Ключевые слова: аморфные тела, упругие свойства, случайные матрицы, нанокомпозиты.

DOI: 10.21883/FTT.2019.07.47846.402

\section{1. Введение}

Одним из самых востребованных разделов современной теории конденсированных сред является физика наноструктур и нанокомпозитов, как с точки зрения теории, так и их применения в различных прикладных областях. Установлено, что внедрение наночастиц в полимерную матрицу даже в небольших концентрациях может приводить к существенному изменению ее упругих свойств. В работах [1,2] показано, что добавление $3-5 \%$ массовой доли наночастиц может увеличивать упругие модули нанокомпозита на 20-25\%.

При деформации такой системы существенное влияние на ее макроскопическую жесткость оказывает микроскопическая неаффиность смещений [3], поскольку их характерный масштаб оценивается как десятки межатомных расстояний [4]. Поэтому частицы нанометрового размера должны оказывать наиболее существенное влияние на макроскопическую жесткость аморфных и полимерных матриц. В настоящей работе изучается влияние таких нановключений на макроскопическую жесткость аморфной среды. Попутно с этим решается более общая задача о теоретическом нахождении модуля Юнга аморфной системы. При этом применяются методы, основанные на использовании случайных матриц [5].

Работа имеет следующую структуру. В разделе 2 описано построение аморфного тела в рамках модели случайных матриц. В разделах 3 и 4 рассмотрен вопрос об определении модуля Юнга аморфной системы. В разделах 5 и 6 описано добавление нановключений в исходную аморфную матрицу и их влияние на макроскопическую жесткость среды.

\section{2. Модель случайных матриц}

Вблизи положения устойчивого равновесия энергия взаимодействия частиц системы представима в виде суммы некоторых квадратичных форм [6]:

$$
U=\frac{1}{2} \sum_{k}\left(\sum_{i} A_{i k} u_{i}\right)^{2} .
$$

Здесь $u_{i}$ - смещение атома с номером $i$ от положения равновесия, индекс $k$ нумерует „связи“ частиц в системе, энергия которых имеет вид положительно определенной квадратичной формы с коэффициентами $A_{i k}$. Для простоты мы рассматриваем так называемую скалярную модель, в которой смещения атомов $u_{i}$ являются скалярами.

Динамическая матрица $\widehat{M}$, соответствующая матрице силовых констант [7] и описывающая колебания атомов, связана с энергией всей системы следующим образом:

$$
M_{i j}=\frac{1}{\sqrt{m_{i} m_{j}}} \frac{\partial^{2} U}{\partial u_{i} \partial u_{j}} .
$$


С учетом этих формул в случае единичных масс частиц динамическая матрица $\widehat{M}$ имеет вид

$$
\widehat{M}=\widehat{A} \widehat{A}^{T} .
$$

Из этого представления видно, что $\widehat{M}$ удовлетворяет требованию механической устойчивости, то есть все ее собственные числа, соответствующие квадратам собственных частот [3], неотрицательны. Но существуют и другие определенные общие физические свойства колебательной системы, которые накладывают на структуру матрицы $\widehat{M}$ дополнительные ограничения. В аморфных телах, вследствие локального беспорядка, $\widehat{M}$ до некоторой степени носит элемент случайности. Также $\widehat{M}$ должна обладать условием трансляционной инвариантности, которое означает, что сдвиг всей системы как целого не меняет ее потенциальной энергии:

$$
\sum_{j} M_{i j}=\sum_{i} M_{i j}=0 .
$$

Для выполнения этих условий рассмотрим матрицу $\widehat{A}$ как случайную квадратную матрицу, построенную на простой кубической решетке с гауссовым распределением недиагональных матричных элементов, соответствующих взаимодействию между ближайшими соседями [5]. Диагональные элементы при этом равны взятой со знаком минус сумме недиагональных элементов соответствующего столбца матрицы $\widehat{A}$. Это приводит к условию $\sum_{i} A_{i j}=0$, что обеспечивает выполнение важного условия трансляционной инвариантности (4).

Оказывается, что такое построение динамической матрицы $\widehat{M}$ дает нулевую макроскопическую жесткость всей системы [5]. Это связано с тем, что число степеней свободы (число строк $\widehat{A}$ ) равно числу связей (число столбцов $\widehat{A}$ ), так какб по описанному выше построениюб матрица $\widehat{A}$ квадратная [6]. Чтобы модуль Юнга системы был отличен от нуляб число связей должно быть больше числа степеней свободы. Для этого можно, например, увеличить число связей атомов путем добавления новых связей

$$
\widehat{M}=\widehat{A} \widehat{A}^{T}+\mu \widehat{M}_{0}
$$

Стандартная кристаллическая динамическая матрица $\widehat{M}_{0}$ построена на той же решетке, с единичными пружинками между соседними узлами решетки. Параметр системы $\mu$ характеризует степень беспорядка и меняется в интервале $0 \leq \mu<\infty$. Случай $\mu \gg 1$ соответствует кристаллической системе с небольшими флуктуациями связей. Но наибольший интерес представляет случай $\mu \ll 1$, который соответствует аморфному телу с большими флуктуациями.

Представленный выше способ построения динамической матрицы не является единственно возможным, однако, как было показано, разные способы построения $\widehat{M}$ приводят к результатам, которые обладают многими общими свойствами [6].

\section{3. Деформация периодической системы}

Для нахождения модуля Юнга системы, описанной с помощью модели случайных матриц, необходимо сначала деформировать систему, а затем искать минимум ее потенциальной энергии, который соответствует положению равновесия. Как было отмечено в предыдущем разделе, модуль Юнга зависит от соотношения числа столбцов и строк матрицы $\widehat{A}$. Будем считать, что $\widehat{A}$ может быть любого вида (прямоугольной или квадратной), в общем случае с размерами $N \times K$.

Рассмотрим случай бесконечной периодической системы, периодическая ячейка которой имеет вид куба со стороной $L$ и содержит достаточно большое число атомов $N \gg 1$. Ячейки образуют простую кубическую решетку, каждая ячейка имеет свой уникальный номер $\mathbf{n}=\left(n_{x}, n_{y}, n_{z}\right)$. Каждый атом в такой системе описывается номером ячейки $\mathbf{n}$, номером атома внутри ячейки $i$ и смещением $u_{i}^{\mathbf{n}}$. Аналогичным образом связи тоже приобретают номер ячейки $\mathbf{p}=\left(p_{x}, p_{y}, p_{z}\right)$ и уникальный номер внутри ячейки $k$. Тогда элементами матрицы $\widehat{A}$ являются $A_{i k}^{\mathbf{n}, \mathbf{p}}$. Поскольку система периодична, то $A_{i k}^{\mathbf{n}, \mathbf{p}}$ зависит от разницы координат ячеек $\mathbf{n}-\mathbf{p}$, что можно записать с помощью одного верхнего индекса $\mathbf{m}=\mathbf{n}-\mathbf{p}$ :

$$
A_{i k}^{\mathbf{n}, \mathbf{p}}=A_{i k}^{\mathbf{n}-\mathbf{p}}=A_{i k}^{\mathbf{m}} .
$$

Теперь приложим некоторую деформацию $\varepsilon=\left(\varepsilon_{x}, \varepsilon_{y}, \varepsilon_{z}\right)$ к системе (в скалярной модели деформация описывается вектором). В состоянии равновесия внутренние деформации всех периодических ячеек одинаковы, поэтому они приобретают новые размеры $\left(L+\varepsilon_{x} L\right) \times\left(L+\varepsilon_{y} L\right) \times\left(L+\varepsilon_{z} L\right)$. В силу периодических условий смещения атомов можно разложить на аффинную и неаффинную компоненты

$$
u_{i}^{\mathrm{n}}=u_{i, \mathbf{n}}^{\mathrm{aff}}+\widetilde{u}_{i} .
$$

Аффинная компонента смещения атома имеет вид

$$
u_{i, \mathbf{n}}^{\mathrm{aff}}=\boldsymbol{\varepsilon} \cdot\left(\mathbf{R}_{i}+L \mathbf{n}\right),
$$

где $\mathbf{R}_{i}+L \mathbf{n}-$ положение равновесия $i$-го атома с начальной координатой $\mathbf{R}_{i}$ в ячейке с номером $\mathbf{n}$. Неаффинное смещение $\widetilde{u}_{i}$ не зависит от номера ячейки $\mathbf{n}$.

Для поиска равновесного положения деформированной системы необходимо найти такие смещения $\widetilde{u}_{i}$, которые минимизируют полную потенциальную энергию $U$. Поскольку полная энергия системы, состоящей из бесконечного числа одинаково деформированных ячеек, расходится, то рассмотрим энергию только одной ячейки. В соответствии с формулой (1):

$$
U_{1}=\frac{1}{2} \sum_{k}\left(\sum_{i, \mathbf{m}} A_{i k}^{\mathrm{m}}\left(\widetilde{u}_{i}+u_{i, \mathbf{m}}^{\mathrm{aff}}\right)\right)^{2} .
$$


Введя новые обозначения, энергию можно представить в виде

$$
U_{1}=\frac{1}{2} \sum_{k}\left(\sum_{i} A_{i k} \widetilde{u}_{i}-b_{k}\right)^{2}
$$

где

$$
\begin{gathered}
A_{i k}=\sum_{\mathbf{m}} A_{i k}^{\mathbf{m}}, \\
b_{k}=-\sum_{i, \mathbf{m}} A_{i k}^{\mathbf{m}} u_{i, \mathbf{m}}^{\text {aff }} .
\end{gathered}
$$

\section{4. Определение модуля Юнга}

Равновесное положение атомов определяется путем минимизации энергии $U_{1}$ по всем возможным неаффинным деформациям $\widetilde{u}$. Соответствующий минимум энергии определяет значение модуля Юнга $E$ следующим образом:

$$
E=\frac{2}{L^{3} \varepsilon^{2}} \min _{\tilde{u}} U_{1}
$$

где $L^{3}$ - объем периодической ячейки. Рассматриваемая нами периодическая ячейка содержит большое число атомов и описывает аморфное тело, поэтому минимальное значение $U_{1}$ не зависит от направления деформации $\varepsilon$, в результате чего $\min _{\tilde{u}} U_{1} \sim \mathcal{\varepsilon}^{2}$ и модуль Юнга не зависит от деформации.

Согласно формуле (10), энергия $U_{1}$ имеет вид суммы квадратичных форм, поэтому минимальное значение $U_{1}$ можно оценить в рамках метода наименьших квадратов. Заметим, что минимальное значение $U_{1}$ не зависит от произвольного ортогонального преобразования

$$
\widehat{A}^{\prime}=\widehat{V} \widehat{A} \widehat{W}, \quad \mathbf{b}^{\prime}=\widehat{V} \mathbf{b},
$$

где $\widehat{V}$ и $\widehat{W}-$ некоторые ортогональные матрицы. Если считать, что $\widehat{V}$ и $\widehat{W}$ являются случайными ортогональными матрицами, то матрица $\widehat{A}^{\prime}$ и вектор $\mathbf{b}^{\prime}$ обладают следующими дисперсиями элементов:

$$
\begin{gathered}
\left\langle\left(A_{i j}^{\prime}\right)^{2}\right\rangle=\frac{1}{N K} \sum_{k l}\left(A_{k l}\right)^{2}, \\
\left\langle\left(b_{i}^{\prime}\right)^{2}\right\rangle=\frac{1}{K} \sum_{j}\left(b_{j}\right)^{2}=\sigma_{b}^{2} .
\end{gathered}
$$

Можно предположить, что наиболее важные свойства системы сохранятся, даже если пренебречь корреляциями между элементами как матрицы $\widehat{A}^{\prime}$, так и вектоpa $\mathbf{b}^{\prime}$ [8]. В этом случае можно применить теорему Гаусса-Маркова для определения минимума $U_{1}$ :

$$
\min _{\widetilde{u}} U_{1} \simeq \frac{1}{2}(K-N) \sigma_{b}^{2}
$$

В результате модуль Юнга имеет следующий вид:

$$
E \simeq \frac{(K-N) \sigma_{b}^{2}}{L^{3} \varepsilon^{2}}=\left(\frac{K}{N}-1\right) E_{0} .
$$

Из этой формулы следует, что модуль Юнга $E$ пропорционален разности числа связей (ширина матрицы $\widehat{A})$ и числа степеней свободы (высота матрицы $\widehat{A}$ ).
В частности, при $K \leq N$ модуль Юнга равен нулю. Действительно, когда число связей $K$ не больше числа степеней свободы $N$, то система слишком мягкая и не обладает макроскопической жесткостью. Величину $E_{0}=N \sigma_{b}^{2} / L^{3} \varepsilon^{2}$ назовем локальным модулем Юнга, поскольку эта величина характеризует силу отдельно взятых связей. В рассматриваемой безразмерной модели $E_{0}=2$. Если $K-N \ll N$, то макроскопический модуль Юнга $E$ много меньше локального модуля Юнга $E_{0}$.

В следующем разделе в нашу аморфную среду будем добавлять жесткие нановключения. Такие добавки меняют число связей и число степеней свободы системы, тем самым внося дополнительный вклад в общую жесткость среды.

\section{5. Добавление нановключений}

Большой интерес представляет влияние жестких нановключений на упругие свойства более мягкой полимерной или аморфной матрицы. Для простоты будем считать, что нановключения не деформируются. Тогда процедура добавления жестких наночастиц может быть выполнена следующим образом.

Рассмотрим описанное ранее аморфное тело, которое состоит из больших периодических ячеек $L \times L \times L$ и описывается матрицей $\widehat{A}$. В каждую ячейку добавим $N_{\text {inc }}$ нановключений с радиусом $R$ и случайными координатами $\mathbf{R}_{h}^{\text {inc }}$, индекс $h$ нумерует нановключения и пробегает значения от 1 до $N_{\text {inc. }}$ Будем считать, что атомы, лежащие в области $\left|\mathbf{R}_{i}-\mathbf{R}_{h}^{\text {inc }}\right|<R$, принадлежат нановключению с номером $h$ и образуют жесткий кластер. Некоторые нановключения могут перекрываться, тогда образуется общий жесткий кластер. Поэтому

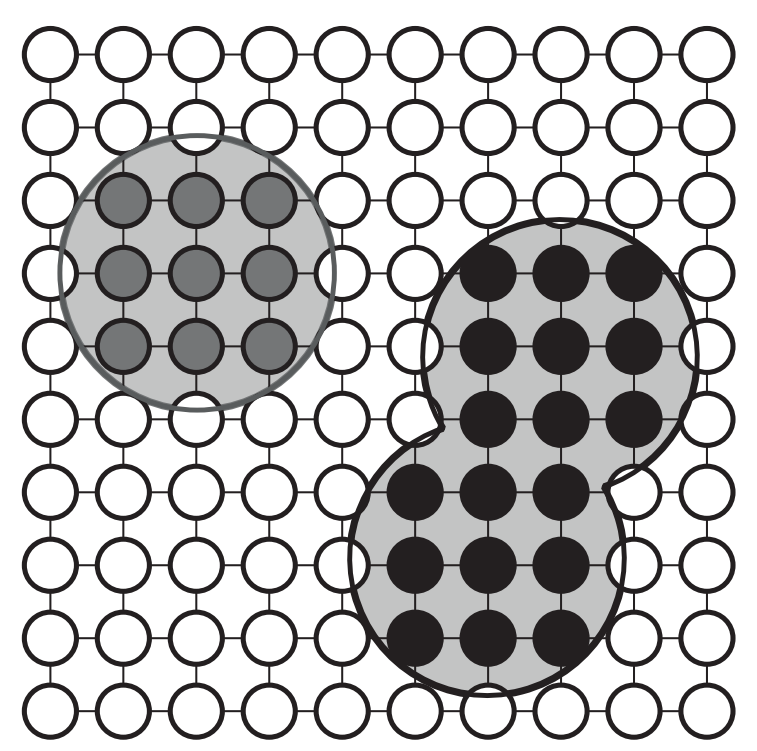

Рис. 1. Модель двумерной решетки аморфного тела размером $10 \times 10$ атомов с нановключениями радиуса $R=1.8$. Белые атомы принадлежат исходной аморфной системе. Серым и черным цветом обозначены атомы, попавшие в разные жесткие кластеры. 


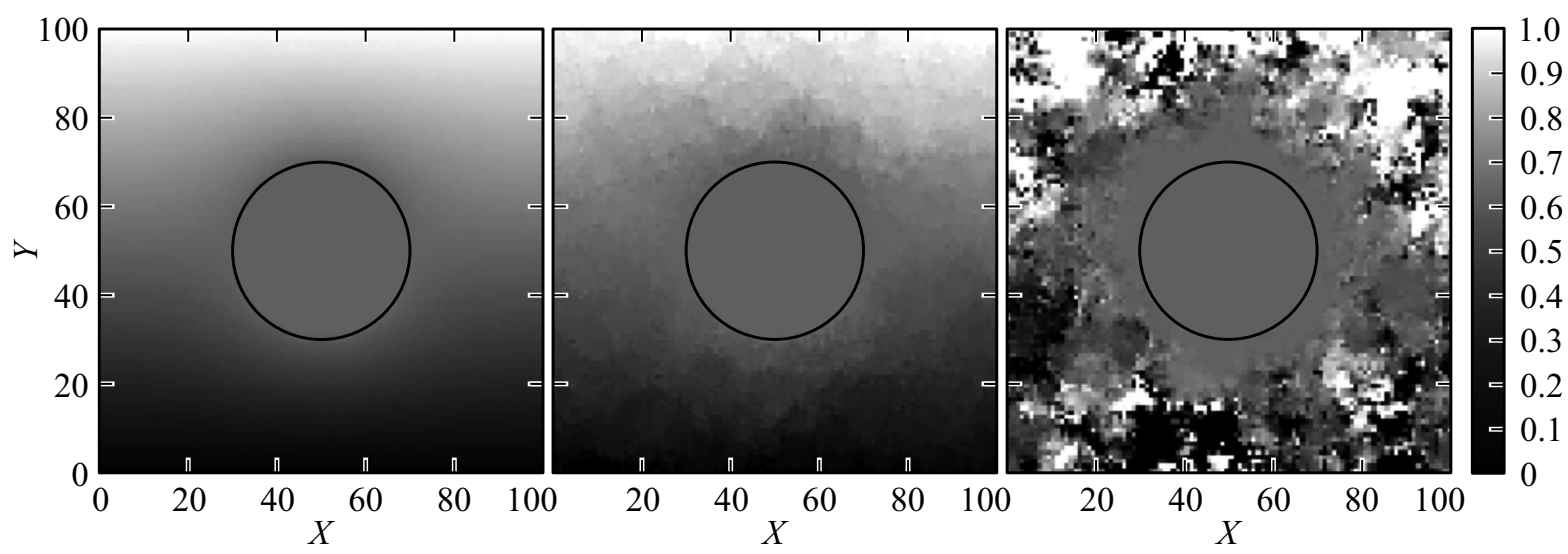

Рис. 2. Смещения атомов двумерного аморфного тела размером $100 \times 100$ с нановключением радиуса $R=20$ для различных параметров системы $\mu$ : на левой панели $\mu=100$, на центральной $-\mu=0.01$, на правой $-\mu=0$. Окружностью обозначена граница нановключения.

число жестких кластеров может быть меньше, чем число нановключений. Так, на рис. 1 добавленные три нановключения образуют два разных жестких кластера. Поскольку мы рассматриваем скалярную модель, каждый жесткий кластер имеет всего одну степень свободы.

После объединения некоторых групп атомов в жесткие кластеры общее число степеней свободы изменилось. Новое число степеней свободы $N_{1}$ можно определить следующим образом:

$$
N_{1}=N-N_{c}+C \text {, }
$$

где $N$ - старое число степеней свободы (полное число атомов), $N_{c}$ - число частиц во всех жестких кластерах, $C$ - количество различных жестких кластеров. Каждая степень свободы в новой системе затрагивает движение одного или нескольких атомов исходной системы. Введем матрицу $\widehat{P}$ с размером $N_{1} \times N$ такую, что $P_{i j}=1$, если степень свободы с номером $i$ в новой системе затрагивает движение атома $j$ в исходной системе (остальные элементы равны нулю). Тогда новая система описывается матрицей силовых констант

$$
\widehat{\Phi}_{1}=\widehat{P} \widehat{M} \widehat{P}^{T}
$$

и динамической матрицей

$$
\widehat{M}_{1}=\widehat{m}_{1}^{-1 / 2} \widehat{\Phi}_{1} \widehat{m}_{1}^{-1 / 2},
$$

где $\widehat{m}_{1}=\widehat{P} \widehat{P}^{T}$ - диагональная матрица масс, которая в новой системе отлична от единичной матрицы. Нетрудно заметить, что новую динамическую матрицу можно представить в виде $\widehat{M}_{1}=\widehat{B} \widehat{B}^{T}$, где $\widehat{B}=\widehat{m}_{1}^{-1 / 2} \widehat{P} \widehat{A}$.

Матрица $\widehat{B}$ имеет размер $N_{1} \times K$. Формально, после объединения атомов в жесткие кластеры число связей в новой системе совпадает с числом связей $K$ в исходной системе. Однако заметим, что в новой системе некоторые связи могут соединять атомы, и так объединенные в один жесткий кластер. Действительно, связь с номером $k$ описывается $k$-ым столбцом в матрице $\widehat{A}$.
Ненулевые элементы данного столбца указывают на то, как взаимодействуют соответствующие атомы между собой. В рассматриваемой модели в каждом столбце исходной матрицы $\widehat{A}$ имеется семь ненулевых элементов: один атом и шесть его ближайших соседей. Если все такие атомы попали в один жесткий кластер, то мы получим полностью нулевой $k$-ый столбец в матрице $\widehat{B}$. Такие тривиальные связи не дают никакого вклада в динамику атомов, поэтому мы можем исключить такие столбцы из дальнейшего рассмотрения. Таким образом, число нетривиальных связей

$$
K_{1}=K-K_{z}
$$

где $K_{z}$ - число нулевых столбцов в матрице $\widehat{B}$.

На рис. 2 изображена двумерная иллюстрация смещений атомов в модели $\widehat{M}=\widehat{A} \widehat{A}^{T}+\mu \widehat{M}_{0}$ при добавлении одного нановключения для различных значений параметра системы $\mu$. Аффинные смещения играют основную роль в системах с $\mu \gg 1$, их градиент при деформации системы отчетливо виден на левой панели. При $\mu \ll 1$ основной является неаффинная компонента смещения, имеется сильный разброс смещений вокруг нановключения.

\section{6. Жесткость системы с нановключениями}

Добавление нановключений приводит к изменению модуля Юнга $\Delta E$, которое зависит от концентрации нановключений $n$ и их радиуса $R$. Из макроскопической теории упругости следует, что $\Delta E \sim n R^{3}$. Однако, как уже было отмечено, на малых масштабах $R$ эта теория неприменима. В соответствии с формулой (18), модуль Юнга исходной системы $E \sim(K-N)$, модуль Юнга системы с нановключениями $E_{1} \sim\left(K_{1}-N_{1}\right)$. Тогда, согласно формулам (19) и (20), изменение жесткости 


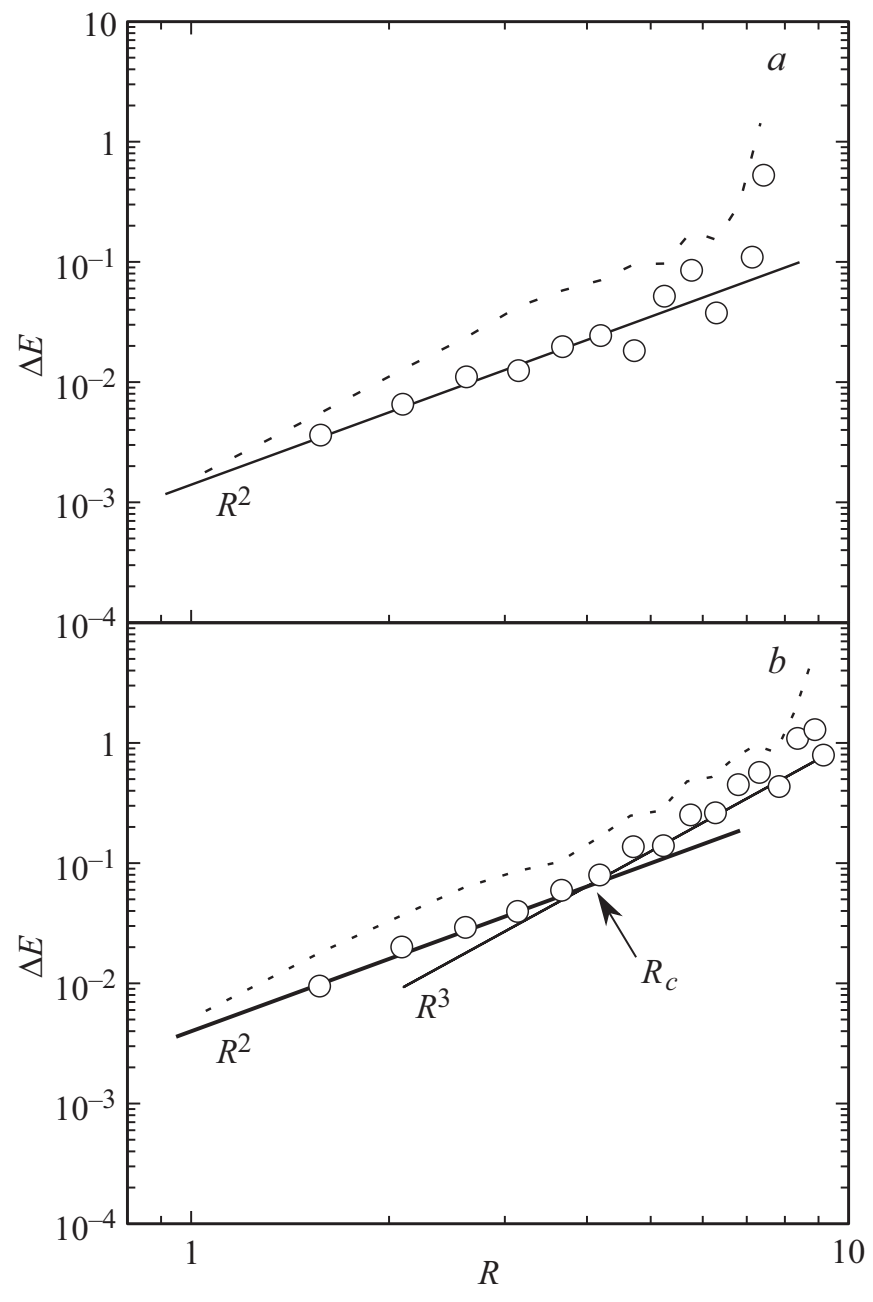

Рис. 3. Зависимость изменения модуля Юнга от радиуса нановключений при их концентрации $n=0.2 \%$ в объеме системы $30 \times 30 \times 30$ для различных параметров $\mu$ : на рисунке $(a)$ $\mu=0$, на рисунке $(b) \mu=0.001$. Кружками обозначен численный расчет, пунктирная кривая - теоретический результат, формула (18). Расчет выполнен в безразмерной модели, где за единицу длины принята постоянная решетки $a_{0}$.

$\Delta E=E_{1}-E$ будет иметь вид

$$
\Delta E \simeq \frac{N_{c}-C-K_{z}}{N} E_{0} .
$$

Число частиц во всех кластерах $N_{c}$ пропорционально всему объему нановключений. Если исключить из этого объема внутренние атомы $K_{z}$, то останутся только поверхностные атомы, лежащие на сфере нановключения радиусом $R$. Другими словами, из формулы (23) следует, что $\Delta E \sim n R^{2} a_{0} E_{0}$, где $a_{0}$ - межатомное расстояние.

На рис. 3 представлен численный расчет изменения модуля Юнга $\Delta E$ системы с нановключениями в зависимости от их радиуса $R$ для модели случайных матриц (5). Расчет сделан согласно формуле (10) путем метода наименьших квадратов. Видно, что в случае $\mu=0$ (рис. 3, a) $\Delta E \sim R^{2}$, и формула (18) хорошо согласуется с численным расчетом. В случае $\mu \neq 0$ (рис. $3, b$ ) на малых масштабах радиуса нановключений зависимость по-прежнему квадратичная, но по мере его увеличения зависимость становится кубической, и в соответствии с макроскопической теорией упругости $\Delta E \sim n R^{3} E$. При определенном радиусе $R=R_{c} \sim a_{0} E_{0} / E$ приведенные выше асимптотические выражения становятся равными между собой. Таким образом, радиус $R_{c}$ определяет границу применимости макроскопической теории упругости и характеризует неоднородность аморфного тела.

\section{7. Заключение}

В настоящей работе изучено влияние нановключений на общую жесткость аморфной или полимерной системы, описываемой с помощью методов случайных матриц. На том масштабе радиусов нановключений, на котором макроскопическая теория упругости неприменима, была установлена квадратичная зависимость изменения модуля Юнга от радиуса нановключений, в то время как в классическом результате зависимость кубическая. Этот масштаб характеризует микроскопическую неоднородность аморфной среды. Формула для модуля Юнга (18) хорошо описывает модель с большими флуктуациями связей, а также случай малых радиусов для отличных от нуля параметров системы $\mu$. Найденная оценка жесткости системы является в некотором смысле универсальным результатом и может быть применена к любым видоизмененным моделям [6] по отношению к (3).

\section{Финансирование}

Работа выполнена при поддержке Российского Научного Фонда (проект номер 17-72-20201).

\section{Конфликт интересов}

Авторы заявляют, что у них нет конфликта интересов.

\section{Список литературы}

[1] D. Stojanovic, A. Orlovic, S. Markovic, V. Radmilovic, P.S. Uskokovic, R. Aleksic. J. Mater Sci 44, 6223 (2009).

[2] О.А. Москалюк, А.М. Самсонов, И.В. Семенова, В.Е. Смирнова, В.Е. Юдин. ЖТФ 87, 266 (2017).

[3] Я.М. Бельтюков, Д.А. Паршин. Письма в ЖЭТФ 93, 660 (2011).

[4] F. Leonforte, R. Boissiere, A. Tanguy, J.P. Wittmer, J-L. Barrat. Phys. Rev. B 72, 224206 (2005).

[5] Y.M. Beltukov, V.I. Kozub, D.A. Parshin. Phys. Rev. B 87, 134203 (2013).

[6] Я.М. Бельтюков, Д.А. Паршин. Письма в ЖЭТФ 104, 570 (2016)

[7] А. Марадулин, Э. Монтролл, Дж. Вейсс. Динамическая теория кристаллической решетки в гармоническом приближении. Мир, М. (1965). С. 383.

[8] Я.М. Бельтюков. Письма в ЖЭТФ 101, 377 (2015).

Редактор Е.Ю. Флегонтова 